\title{
The magnetic field structure of the Small Magellanic Cloud
}

\author{
Antonio Mário Magalhães ${ }^{1}$, Aiara Lobo Gomes ${ }^{1}$, \\ Aline de Almeida Vidotto ${ }^{1}$, Cláudia Vilega Rodrigues ${ }^{2}$, \\ Antonio Pereyra ${ }^{3}$, John Wisniewski ${ }^{4}$, Karen Bjorkman ${ }^{5}$, \\ Jon Bjorkman ${ }^{5}$, Marilyn Meade ${ }^{6}$ and Brian L. Babler ${ }^{6}$ \\ ${ }^{1}$ Depto. de Astronomia, IAG, Universidade de São Paulo, Rua do Matão 1226, São Paulo, \\ SP 05508-900, Brazil \\ email: mario@astro.iag.usp.br \\ ${ }^{2}$ INPE/DAS, S.J. dos Campos, SP, Brazil \\ ${ }^{3} \mathrm{ON} / \mathrm{CNPq}$, Brazil \\ ${ }^{4}$ University of Washington, USA \\ ${ }^{5}$ University of Toledo, USA \\ ${ }^{6}$ University of Wisconsin-Madison, USA
}

\begin{abstract}
We describe two studies of the interstellar magnetic field in regions of the Small Magellanic Cloud (SMC), including those affected by the interaction with the Large Magellanic Cloud (LMC). We use optical polarization data from aligned grains in the interstellar medium of the SMC in order to map the sky-projected direction of the magnetic field and determine characteristics of the SMC and Pan-Magellanic field structures. The earlier, photoelectric data are reanalyzed and they provide values for the average projected magnetic field intensity $(1.7 \times$ $10^{-6} \mathrm{G}$ ) and the random field component intensity $\left(3.5 \times 10^{-6} \mathrm{G}\right)$. Another on-going program uses imaging data and, when concluded, will allow more local estimates of the field intensity in the SMC NE/Wing regions. Additional goals include cross-correlating our field mapping results with those of point sources and structures found by the Spitzer Space Telescope in the SMC between 3.6 and $8 \mu \mathrm{m}$.
\end{abstract}

Keywords. polarization, techniques: polarimetric, galaxies: individual (SMC), Magellanic Clouds, galaxies: magnetic fields

\section{Introduction}

The Magellanic Clouds form with the Galaxy a triple system in continuous interaction. When of the last approach, for instance, the SMC reached $2-5 \mathrm{kpc}$ from the LMC, about 0.2 Gyr ago (Westerlund 1993). These interactions have had great influence on the Clouds. For example, the SMC may have been "stretched" in its Northeast and Wing sections towards the LMC during this last collision. The H I bridge and $\mathrm{H} \alpha$ nebulosities are other effects of such past collisions (Sofue 1994).

Magnetic fields are thought to be strong enough to influence the gas dynamics in galaxies (Zweibel \& Heiles 1997; Beck 2008). Our on-going project aims at studying the interstellar magnetic field in these SMC regions affected by the collisions. We employ the technique of optical imaging polarimetry, detecting the polarization caused by magnetically aligned grains in the SMC. 


\section{Interstellar polarization}

Interstellar grains can be partially aligned in the magnetic field of the interstellar medium (Davis \& Greenstein 1951; Hoang \& Lazarian 2008). The detailed mechanism is still being actively discussed, with radiative torque receiving support lately (Hoang \& Lazarian 2008). Regardless of the theory, the mechanism is expected to first align the small axis of the grain with its angular momentum, or spin, axis and then align the spin axis with the magnetic field. The end result has the polarizing grains rotating with their longest profile perpendicular to the magnetic field. Starlight shining through such grains will then have the transmitted electric field vibrations predominantly along the sky-projected magnetic field direction.

Starlight polarization is hence a direct tracer of the ISM magnetic field projected on the sky. This technique has been used in the optical to map Galactic fields in dark clouds (e.g., Pereyra \& Magalhães 2004) and in shocked regions (Pereyra \& Magalhães 2007). It can also be used to probe the magnetic field correlation length in the Galaxy's ISM (Magalhães et al. 2005). On a Galactic scale, Heiles (1996) and Fosalba et al. (2002) have used the compiled data for the Galaxy in order to probe from the field structure in the Solar neighborhood to the ratio of random to uniform components of the field.

Observed linear polarization values towards the general ISM in the Galaxy are typically of a few percent, although higher values ( $\sim 10-15 \%$ or so) may be observed towards regions where the magnetic field geometry is favourable (Pereyra \& Magalhães 2004, 2007). In the SMC case however, in part because of the small dust-to-gas ratio and in part to our incomplete knowledge, the reported values of interstellar polarization are in general considerably smaller (Rodrigues et al. 1997; Wisniewski et al. 2007a; section 3).

\section{Interstellar polarization in the $\mathrm{SMC}$}

Mathewson \& Ford (1970) and Schmidt (1976) provided the first measurements of starlight polarization for SMC members. These works and the first photoelectric measurements of Magalhães et al. (1990) have suggested a magnetic field aligned with the H I bridge (McGee \& Newton 1981, 1986). More recently, Mao et al. (2008) have reanalyzed the Mathewson \& Ford (1970) data and, together with radio Faraday rotation data, concluded that the overall magnetic field of the SMC may indeed lie along the line connecting the centers of each Cloud.

Here we re-discuss the Magalhães et al. (1990) data, the details of which we expect to publish shortly (Magalhães et al., in preparation). We also follow the practise of separating the SMC main body and NE/Wing into 5 sections (I through V; Schmidt 1976; Rodrigues et al. 1997). In particular, we use the corresponding five Galactic foreground correction values of Rodrigues et al. (1997), in their study of SMC dust (their Table 2). Since the SMC interstellar polarization values are rather small (typically $\sim 1 \%$ or smaller), this correction is crucial. The observational uncertainty in each data value is added quadratically to that of the foreground correction.

The total sample has 132 SMC stars distributed along its main body and NE/Wing sections. After correcting for the Galactic foreground values and selecting only objects with SMC polarization $P$ and error $\sigma_{\mathrm{P}}$ such that $P / \sigma_{\mathrm{P}} \geqslant 2,66$ objects remain. We then transform their equatorial position angle to a "Magellanic" position angle, $\Theta_{\mathrm{M}}$, such that this new angle is zero along the SMC-LMC direction (Schmidt 1976, Mao et al. 2008).

Fig. 1 shows the distribution of the $\Theta_{\mathrm{M}}$ values for the whole sample. Clustering around two position angle values, $\Theta_{\mathrm{M}} \sim 0^{\circ}$ and $\sim 60^{\circ}$, can be noted. In other words, one of the tendencies is for the field to indeed lie along the SMC-LMC direction, and another 


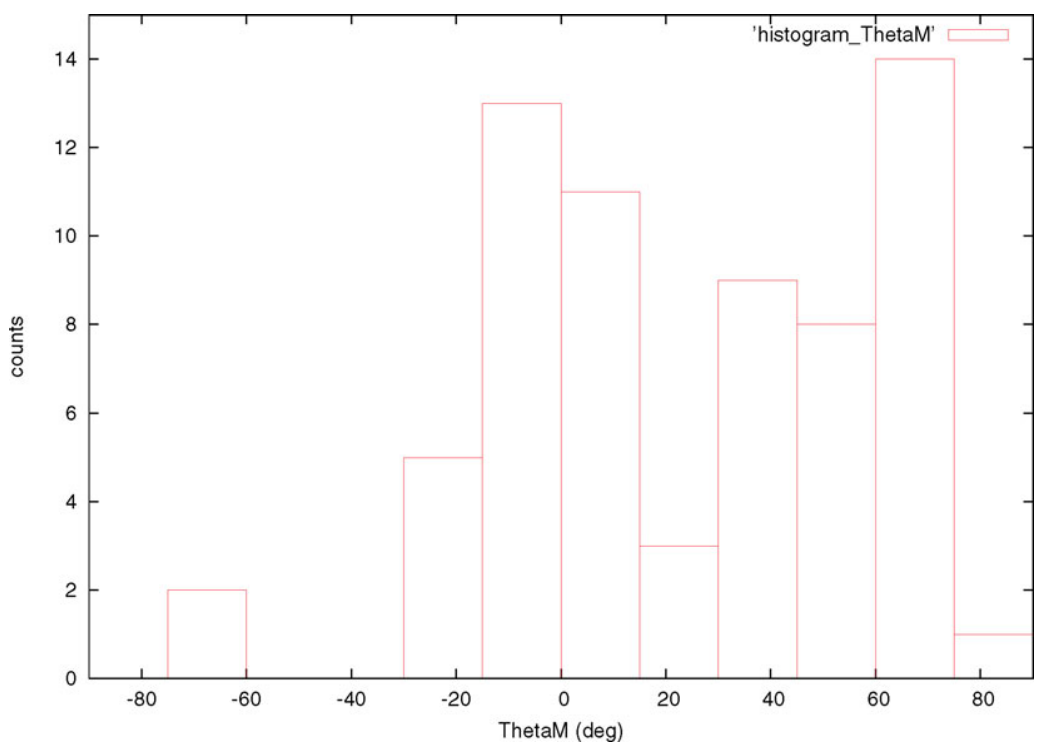

Figure 1. Histogram of position angles of optical polarization vectors across the SMC (main body and NE/Wing sections combined), using the data of Magalhães et al. (1990). $\Theta_{\mathrm{M}}=0$ along the SMC-LMC direction.

one represent a tendency of the field to roughly lie along the N-S direction. The latter is because the average correction from the equatorial to the Magellanic position angle for the main body is $\sim 113^{\circ}$, giving almost a N-S direction (i.e., $0^{\circ} \equiv 180^{\circ}$ ).

These two tendencies are confirmed if we examine separately the histograms of $\Theta_{M}$ for regions I through III (main body) and IV-V (NE and Wing sections). Fig. 2 shows the histogram across the main body and the values again cluster around $\Theta_{\mathrm{M}} \sim 0^{\circ}$ and $\sim 60^{\circ}$, albeit with a less marked tendency. Fig. 3, for the NE+Wing sections, shows again the two tendencies but this time more clearly. This may be understood if we remember that lines of sight through the main body are more likely to cross higher density regions that those in the NE/Wing sections; higher densities will tend to drag the field around compared to less dense regions and there should be a broader dispersion of polarization values along the line of sight. This is consistent with the fact that the SMC is more distant than the LMC and the bridge is seen 'pointing' to the observer; the observed polarization is mostly produced in these layers lying in front of the SMC (Magalhães et al. 1990).

The distribution of position angles allows us to estimate the component of the magnetic field intensity in the plane of the sky using the formulation of Falceta-Gonçalves et al. (2008). We first estimate the random component of the magnetic field, $\delta B$, using their eq. 7 , which equates the random thermal and magnetic energies. Using the SMC H I velocity dispersion (22 $\pm 2 \mathrm{~km} \mathrm{~s}^{-1}$; Stanimirović et al. 2004) and density $\left(n_{\mathrm{H}} \sim 0.1 \mathrm{~cm}^{-3}\right.$, Mao et al. 2008), we find $\delta B \approx 3.5 \times 10^{-5} \mathrm{G}$. This values compares well with the estimate of Mao et al. (2008) using synchrotron emission.

Now, eq. 9 of Falceta-Gonçalves et al. (2008) provides a modified form for the Chandrasekhar-Fermi relation between the dispersion in position angles and the total field intensity projected on the sky, $B_{\mathrm{sky}}^{\mathrm{u}}+\delta B$ (which they call $B_{\mathrm{sky}}^{\mathrm{ext}}+\delta B$ ). Essentially, for a given random velocity dispersion a narrow dispersion in $\Theta_{M}$ implies a larger skyprojected, uniform field intensity, as physically expected. Using this equation, we obtain $B_{\text {sky }}^{\mathrm{u}} \approx 1.7 \times 10^{-6} \mathrm{G}$. This is to be compared to $(1.6 \pm 0.4) \times 10^{-6} \mathrm{G}$ obtained by Mao 


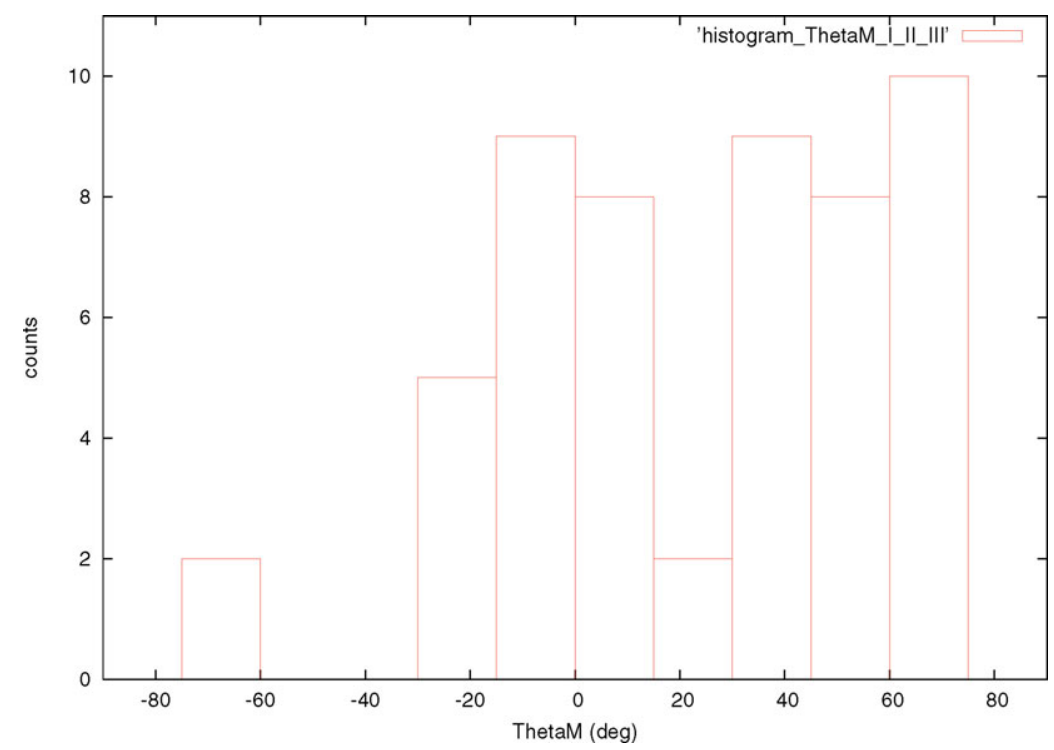

Figure 2. Same as Fig. 1 but for the SMC main body only (regions I-III of Schmidt 1976).

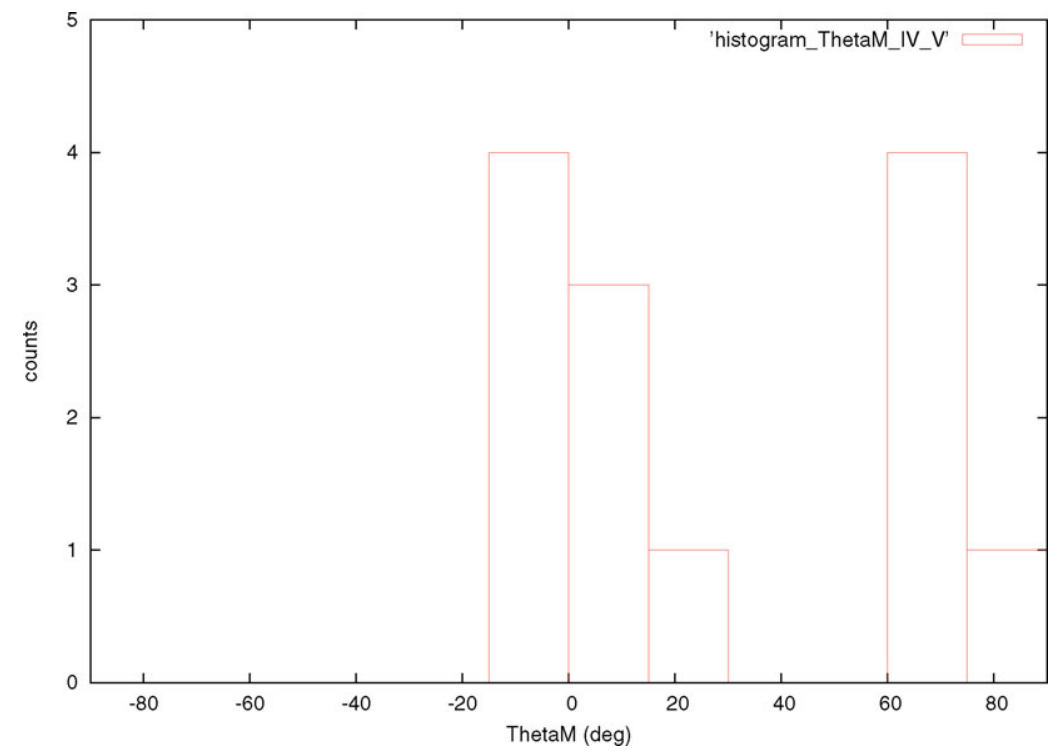

Figure 3. Same as Fig. 1 but for the SMC NE and Wing sections only (regions IV and V of Schmidt 1976).

et al. (2008) using the Mathewson \& Ford (1970) sample. The formal uncertainty in our result is not too bad $(\sim 20 \%)$ but the real answer, given the uncertainty in the density, for instance, is probably within a factor of a few.

The above discussion concerned the overall magnetic field of the SMC. We (Wisniewski et al. 2007a,b) have observed 6 LMC and 6 SMC open clusters for studying Be star disks in low metallicity environments. These observations were used to derive the foreground values of the interstellar polarization within each Cloud (after Galactic foreground subtraction). The SMC interstellar polarization values and their position angles towards the six directions observed are consistent with the sample discussed above. However, in the 


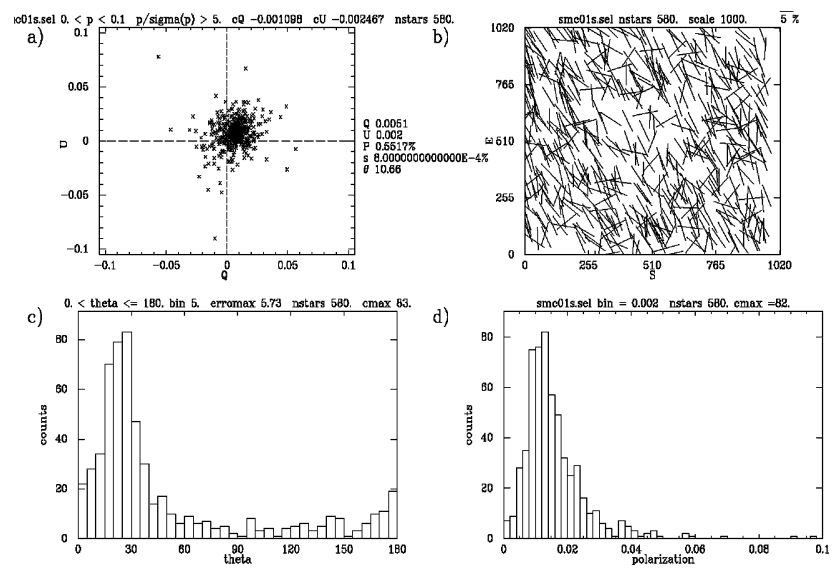

Figure 4. Foreground corrected, V-band imaging polarization data for a $8^{\prime} \times 8^{\prime}$ field towards $\left[1^{\mathrm{h}} 00^{\mathrm{m}},-72^{\circ} 00^{\prime}\right.$ (eq. 1975)], in the SMC NE region. Stars for which $\mathrm{P} / \sigma_{P} \geqslant 7$ have been selected. From left to right and top to bottom: Q-U diagram, position angle vector diagram, histogram of equatorial position angles and histogram of polarization values.

denser region towards NGC 2100, in the LMC 2 Supershell, Wisniewski et al. (2007b) have found more complex morphology, consistent with the fact that, in the main body of the SMC, we see a larger spread of position angles discussed earlier in this section.

\section{Imaging polarimetry program in the NE/Wing of the SMC}

We are currently performing an analysis of CCD optical imaging polarimetry data towards several (currently 28) directions spread along the NE and Wing sections of the SMC. Each CCD field is $8^{\prime} \times 8^{\prime}$ and the images were taken through the $V$ filter. Fig. 4 shows an example of the results for a field centered at about $\left[1^{\mathrm{h}} 00^{\mathrm{m}},-72^{\circ} 00^{\prime}\right.$ (eq. 1975)].

For this field, Fig. 4 shows that there were more than 480 objects with $P / \sigma_{\mathrm{P}} \geqslant 7$. The histogram of position angles shows a single prevalent tendency towards that part of the SMC. The polarization value histogram also shows that many objects with $P \gtrsim 2 \%$ have been found. All of this shows that the data have a potential to provide estimates of the magnetic field towards several directions across the SMC, allowing for a study of the magnetic field intensity as a function of the environment. The combination of the polarization data with other types of data (such as visual absorption) should provide further quantities of interest, such as $P_{\mathrm{V}} / A_{\mathrm{V}}$, which measures the alignment efficiency.

The Spitzer Space Telescope is providing data on the SMC that will have a direct bearing on such polarization data. The programs $\mathrm{S}^{3} \mathrm{MC}$ (Bolatto et al. 2007) and SAGE-SMC (Gordon et al., this volume) are very good examples. For instance, the study of how the magnetic field structures correlates with the wealth of structures seen by Spitzer should provide information on the physical processes in the SMC interstellar medium. Also, Spitzer data on the dust along the SMC Wing will help interpret the polarization data in that region, where the SMC polarization is expected to be very small, and will provide a check on the accuracy of the Galactic foreground correction. Finally, point source catalogs from Spitzer imagery, in association with our optical polarization, should eventually provide detection of significant intrinsic polarization for some sources, indicative of non-spherically symmetric material around such stars. 


\section{Conclusions}

Starlight polarimetry is a good tool for mapping the magnetic field across the SMC, despite the overall low dust content of that galaxy. The general magnetic field structure of the SMC presents a Pan-Magellanic component towards the Bridge and a main body component, roughly in the N-S direction. The estimated field intensities are $\delta B \approx 3.5 \times$ $10^{-5} \mathrm{G}$ for the random component and $B_{\mathrm{sky}}^{\mathrm{u}} \approx 1.7 \times 10^{-6} \mathrm{G}$ for the uniform, sky-projected component. The on-going analysis of optical imaging polarimetry across the SMC NE and Wing sections should provide a much better understanding of the behaviour of the magnetic field throughout the SMC and its relationship to the ISM of the galaxy.

\section{Acknowledgements}

Research on polarimetry at the Astronomy Department, IAG-USP, is supported by São Paulo state funding agency FAPESP. AMM is also supported by CNPq.

\section{References}

Beck, R. 2008, in F. A. Aharonian, W. Hofmann, \& F. M. Rieger (eds.), High Energy GammaRay Astronomy, AIP Conf. Proc., 1085, p. 83

Bolatto, A. D., Simon, J. D., Stanimirović, S., et al. 2007, ApJ, 655, 212

Davis, L. J. \& Greenstein, J. L. 1951, ApJ, 114, 206

Falceta-Gonçalves, D., Lazarian, A., \& Kowal, G. 2008, ApJ, 679, 537

Fosalba, P., Lazarian, A., Prunet, S., \& Tauber, J. A. 2002, ApJ, 564, 762

Heiles, C. 1996, ApJ, 462, 316

Hoang, T. \& Lazarian, A. 2008, MNRAS 388, 117

Magalhães, A. M., Loiseau, N., Rodrigues, C. V., \& Piirola, V. 1990 in Galactic and intergalactic magnetic fields, IAU Symposium 140 (Dordrecht, The Netherlands: Kluwer Academic Publishers), p. 255

Magalhães, A. M., Pereyra, A., Melgarejo, R., et al. 2005, in A. Adamson, C. Aspin, C. J. Davis, \& T. Fujiyoshi (eds), Astronomical Polarimetry: Current Status and Future Directions, ASP Conference Series 343, p. 305

Mao, S. A., Gaensler, B. M., Stanimirović, S., Haverkorn, M., McClure-Griffiths, N. M., StaveleySmith, L., \& Dickey, J. M. 2008, ApJ, in press, ApJ, 688, 1029

Mathewson, D. S. \& Ford, V. L. 1970, ApJ, 160, L43

McGee, R. X. \& Newton, L. M. 1981, Proceedings of the Astronomical Society of Australia, 4, 189

McGee, R. X. \& Newton, L. M. 1986, Proceedings of the Astronomical Society of Australia, 6, 471

Pereyra, A. \& Magalhães, A. M. 2004, ApJ, 603, 584

Pereyra, A. \& Magalhães, A. M. 2007, ApJ, 662, 1014

Rodrigues, C. V., Magalhães, A. M., Coyne, G. V., \& Piirola, V. 1997, ApJ, 485, 618

Schmidt, T. 1976, A\&AS, 24, 357

Sofue, Y. 1994, PASJ, 46, 431

Stanimirović, S., Staveley-Smith, L., \& Jones, P. A. 2004, ApJ, 604, 176

Westerlund, B. E. 1993, in B. Bacheck (ed.), New Aspects of Magellanic Cloud Research, 416, p. 7

Wisniewski, J. P., Bjorkman, K. S., Magalhães, A. M., Bjorkman, J. E., Meade, M. R., \& Pereyra, A. 2007a, ApJ, 671, 2040

Wisniewski, J. P., Bjorkman, K. S., Magalhães, A. M., \& Pereyra, A. 2007b, ApJ, 664, 296

Zweibel, E. G. \& Heiles, C. 1997, Nature, 385, 131 